\title{
Overview of Diagnostic Reasoning: Hypothetical- deductive Strategy, Problem Representation, Semantic Qualifiers, Illness Scripts, Pattern Recognition and Prototypes
}

\author{
Sekelani S. Banda \\ Department of Medical Education Development, School of Medicine \\ University of Zambia, Lusaka, Zambia \\ P.O Box 50110,Lusaka, Zambia, E-mail: ssbanda@zamnet.zm
}

\begin{abstract}
Context: This current paper is a non-systematic review of diagnostic reasoning exclusive of the Bayesian model which we considered a decisionmaking stratagem. The paper reviews primarily the literature on hypothetical-deductive reasoning and pattern recognition, the two archetypes of analytical and non-analytical reasoning, respectively. Though a lot of work has been reported in this field internationally our medical training in Zambia does not overtly tackle diagnostic reasoning and expectedly not many clinicians in Zambia are acquainted with the subject matter. Nevertheless, our clinicians pass on to future generations, by apprenticeship, their diagnostic reasoning processes, but to be effective educators clinicians must understand diagnostic reasoning well.
\end{abstract}

Purpose: The purpose of the current paper is threefold: 1) to share the generally accepted concepts in diagnostic reasoning in order to help clinicians become more effective educators in the clinical settings, 2) provoke clinicians and students alike to critically consider the subject of diagnostic reasoning and 3) also to inform readers about prospective research initiatives at the University which intend to investigate diagnostic reasoning practices of Zambian-educated medical graduates.

Conclusions: Generally, there are three accepted diagnostic reasoning models, i.e., hypotheticaldeductive reasoning, pattern recognition, and pathognomonic recognition of signs and symptoms. These are categorised as analytical methods (principally the hypothetical-deductive strategy) and non-analytical methods (pattern recognition and pathognomonic approaches). The Diagnostic Reasoning Research - Zambia Project (DRR-Z) will be the first in Zambia to study diagnostic reasoning processes of graduates from University of Zambia School of Medicine. Clinicians who are well informed about the diagnostic reasoning process can teach it more effectively.

\section{INTRODUCTION}

This current paper presents an overview of diagnostic reasoning and focuses on the hypothetical-deductive model ${ }^{1,2,3,4,5,6,7}$ and the semantic qualifier approach of problem representation, 2, 8, 9. We reviewed over 100 publications on diagnostic reasoning cited on PubMed and African Journals on Line (AJOL) searchable engines and 3 selected textbooks. Three works, which were regarded to be seminal in their fields, served as our principal reviews: one on cognitive psychology; one on clinical problemsolving, and a synopsis of semantic qualifiers, problem representation and synopsis' However, works of many other authors from 1978 to 2009 were also reviewed but our context was limited to the clinical practice of medical doctors. Additionally, the review remained concept-centric rather than author-centric.

Key words: Diagnostic reasoning, hypotheticaldeductive; illness scripts; semantic qualifiers; descriptors; prototypes. 
Experts agree that medical diagnostic reasoning involves data acquisition as the first step and problem representation as the next ${ }^{1,2}$. Researchers have also recognised the hypothetical-deductive reasoning strategy and also identified clinical expertise with problem representation, illness scripts, semantic qualifiers, pattern recognition and patient prototypes $^{1,8}$. These are reviewed in detail.

While the field of diagnostic reasoning has developed internationally and has acquired its own theories based on empirical observations, taxonomies, conceptual frameworks, and theoretical systems, in Zambia it remains largely uncharted. The purpose of the current paper is threefold: 1) to share the generally accepted concepts in diagnostic reasoning in order to help clinicians become more effective educators in the clinical context, 2) provoke clinicians and students alike to critically consider the subject of diagnostic reasoning and 3) inform readers about the pioneering Diagnostic Reasoning Research - Zambia Project (DRR-Z) which will be the first to study diagnostic reasoning processes of graduates from University of Zambia School of Medicine. By studying our uncharted geographical region and locale, the project will assist us to investigate the nature of our local diagnostic reasoning and perhaps propose local conceptual models and generate theories of our own to extend existing international research.

\section{Generally Accepted Concepts of Diagnostic Reasoning}

\section{Hypothetical-Deductive Strategy}

Early work on diagnostic reasoning with hypothetical-deductive models was done by Elstein ${ }^{6}$ in their Medical Inquiry Project. The hypothesis testing approach, however, is much like the "Test Operate Test Exit' (TOTE) programme described by Cohen ${ }^{7}$, which was widely reported by cognitive psychologists researching problem-solving. In hypothetical-deductive process solutions are created by generating a limited number of hypotheses during the initial consideration of a clinical problem and using them to direct further collection of data ${ }^{5,6,10,11}$. If a hypothesis is rejected, an alternative one is considered and treated in the same way ${ }^{511}$. The hypotheses serve as 'organising rubrics in working memory' ${ }^{6}$. Such rubrics help to surmount limited memory capacity and focus problem space that is searched for solutions to a narrow one ${ }^{6}$. This way the hypotheses transform an open-ended medical problem (what is wrong with the patient) into a set of focused enquiries ${ }^{6}$. Elstein ${ }^{6}$ emphasized major steps in diagnostic reasoning: 1) cue acquisition, 2) hypothesis generation, 3) cue interpretation, and 4) hypothesis evaluation or judgement. We consider the Elstein ${ }^{6}$ report a seminal work on hypotheticaldeductive reasoning and many scholars consider it a primary clinical reasoning strategy ${ }^{12,13}$.

However, thoroughness of cue acquisition and accuracy of cue interpretation are independent of each other, 6, 9, 10. Cue acquisition is a function of episodic memory ${ }^{14}$ - memory of regular and routine events - as is the case when medical students work out patients using standard data gathering protocols. The standard protocol usually involves the following steps: 1) rapport, 2) history of present illness, 3) personal and social history, 4) previous medical history, 5) family history and then followed by a physical examination of the body systems, and later laboratory and/or imaging investigations.

The literature suggests that the hypotheticaldeductive method is used when a clinical case is difficult, unfamiliar or the clinician is inexperienced ${ }^{2}$. The analytical process involves searching for and verifying or refuting possibilities ${ }^{3,}$ $10,15,16$. This process starts right from the data acquisition stage and directs further enquiry, physical examination, selection of investigations and probable hypotheses.

The problem-solving fraternity now appears to be agreed that mastery of content supplants problemsolving strategy ${ }^{3,12}$. Accuracy of data interpretation is a function of semantic memory - memory of facts, relationships between signs and symptoms and what they signify ${ }^{14}$. Semantic memory, therefore, deals with the structure, storage, cross-referencing and indexing of knowledge in the human brain ${ }^{1,9}$. How semantic memory relates to diagnostic reasoning is discussed next.

\section{Problem Representation Leading to Pattern Recognition}

Problem representation ${ }^{2,9,16}$ entails the characterisation (or transformation) of a patient's problems into abstract terms. 
Semantic Qualifiers: Bordage ${ }^{1}$ observed that "successful diagnostician use semantic qualifiers more frequently and in more diversified sets in their discourses than diagnosticians who are less successful". Many works ${ }^{9,17,18}$, have demonstrated that expert clinicians used abstract semantic qualifiers to describe clinical cases and that use of such semantic qualifiers was associated with strong clinical reasoning. Semantic qualifiers are descriptors that are usually paired and are used to compare and contrast clinical phenomenon'. Bowen $^{2}$ states that descriptors that are characteristic of the diagnoses are by definition 'defining features' while descriptors that are used for distinguishing the diagnoses from one another are termed 'discriminating features'. Defining and discriminating clinical features of a disease, condition or syndrome are known as "anchor points". The mental action of changing a patients' problems into abstract representation is what helps the retrieval of relevant knowledge from memory ${ }^{9}$. In the transformation process, "terms transform as follows: "Sudden onset of pain" becomes "Acute onset", "I had this problem before" becomes "recurrent", "only one joint is swollen" becomes "monoarticular", for example'. Descriptors are usually paired and used to compare and contrast diagnostic considerations ${ }^{2}$, for example, "multiplesingle", "discrete-continuous", "abrupt-gradual", "severe-mild", "chronic-acute", "infection-noninfectious" 1,2 . It now therefore considered that expert clinicians use abstract semantic qualifiers to describe clinical cases and the use of such semantic qualifiers is associated with strong clinical reasoning $^{9,17,18}$.

Illness Scripts: Because semantic qualifiers are associated to certain diseases, it is widely acknowledged that expert clinicians store and recall diseases, conditions, or syndromes as "illness scripts" ${ }^{15}$. Particular sets of symptoms or phrases become associated with particular diagnoses and over time clinicians develop their own unique repository of patterns based on their existing knowledge and experience ${ }^{11}$. It follows then that expertise in diagnostics varies significantly between individual clinicians and is highly dependent on the clinician's masterly of the particular subject area ${ }^{10}$. The "illness scripts" are connected to semantic qualifiers, and this connection is referred to as problem representation ${ }^{3,10,15,16}$. This relationship between semantic qualifiers and illness scripts is the reason that problem representation activates clinical memory and allows knowledge to become usable for diagnostic reasoning. This being the case, it is expected that in many instances expert clinicians use non-analytical clinical reasoning to merely recognise a set of symptoms and signs and allocate a diagnosis ("pattern recognition") 3, 10,15,16,19.

Prototypes: In prototype theory ${ }^{1}$ medical categories are organised in memory around representative exemplars and these prototypes serve as anchors for other members of the category. This means that when a clinician encounters a case they categorise it into one of their known categories of diseases, e.g., metabolic, infectious, malignancy etc., and then look for a representative disease (prototype) with which to compare the new encounter. Bordage ${ }^{9}$ advocates the teaching of a carefully selected list of categories and prototypes.

Pathognmonic Signs and Symptoms - This applies where a particular finding is a 'signature call' or 'hallmark' of a known diagnosis, e.g. ulnar deviation in rheumatoid arthritis; Kaiser-Fleischer rings in Wilson's disease; blue sclera in osteogenis imperfecta; and the slow relaxing jerks of hypothyroidism ${ }^{2}$. However, scholars consider this rare and of little help in day to day practice ${ }^{2}$.

While hypothetical-deductive, pattern recognition, pathognmonic approaches are generally accepted diagnostic reasoning models the debate on which approach expert clinicians use is ongoing. However, many scholars are becoming persuaded that clinicians approach problems in a flexible manner depending on the perceived disposition of the problem $^{2,3,8,10}$. The familiar and simple cases are solved by pattern recognition; difficult and complex cases are solved by systematic analytical testing of hypotheses ${ }^{10}$. An important contribution to the diagnostic reasoning research could be to check if these approaches and theories apply to doctors/clinicians trained in Zambia.

\section{Future Works}

Diagnostic Reasoning Research - Zambia Project (DRR-Z) which will be the first to study diagnostic reasoning processes of graduates from University of Zambia School of Medicine. By studying our uncharted geographical region, the project will 
assist us to investigate the nature of our local diagnostic reasoning and perhaps propose local conceptual models and generate theories of our own to extend existing international research. A variety of methods have been used in diagnostic reasoning research, for example, 'think aloud protocols', computerised node analysis, and the diagnostic reasoning inventory. Understanding diagnostic reasoning can make clinicians more effective in teaching diagnostic reasoning.

\section{REFERENCES}

1. Bordage, G. (2007). Protypes and Semantic Qualifiers: from past to present. Medical Education; 41:1117-1121.

2. Bowen, J (2006). Educational Strategies to Promote Clinical Diagnostic Reasoning. The New England Journal of Medicine, 355:22172225.

3. Norman, G.R. (2005). Research in Clinical Reasoning: Past history and current trends. Medical Education; 39:98-106.

4. Eva, K.W. (2005). What every teacher needs to know about clinical reasoning. Medical Education; 39: 98-106.

5. Elstein, A.S., Schwartz, A., Higgs, J., Jones, M. (2000). Clinical Reasoning in Medicine. Clinical Reasoning in the Health Professions. Oxford, UK: Butterworth Heinemann: 95-106.

6. Elstein, S.A., Shulman, L.S., Sprafka, S. A. (1978). Medical Problem Solving: An Analysis of Clinical Reasoning. Cambridge, MA: Harvard University Press; 276.

7. Cohen, G. (1977). The Psychology of Cognition. Academic Press. London. ISBN 012-1787750.

8. Norman, G. (2006). Building on Experience The Development of Clinical Reasoning. The New England Journal of Medicine; 355 (21): 2251-2252.

9. Bordage, G. (1999). Why did I miss the diagnosis? Some cognitive explanations and educational implications. Academic Medicine, 75: Suppl: S138-S143.
10. Elstein, A.S., Schwarz, A. (2002). Clinical Problem Solving and Diagnostic Decision Making: Selective Review of the Cognitive Literature. $B M J ; 324: 729-732$.

11. Round, A. (2000). Introduction to clinical $\mathrm{r}$ e a s o n ing. S t u d e n t B M J . Http://student.bmj.com/back_issues/0200/edu cation/15.html 28/11/2008.

12. Bloch, R.F., Hofer, D., Feller, S., and Hodel, M. (2003). The role of strategy and redundancy in diagnostic reasoning. BMC Medicine Education 3: 1, doi:10.1186/1472-6920-3-1.

13. Yoshinori, N., Kunihiko, M., Hiroshi, I., Masatomo, K., and Tuguya, F. (2002). Quantitative Evaluation of the Diagnostic Thinking Process in Medical Students. J Gen Intern Med. 17 (11):848-853.

14. Kurkland, M., and Lupoff, R. (1999). The Complete Idiot's Guide to Improving your Memory. Alpha Books, A Pearson Education Company. Indianapolis. ISBN 0-02862949-3.

15. Schmidt, H.G., Norman, G.R., Boshuizen, H.P. (1990). A congnitive perspective on medical expertise: theory and implications. Academic Medicine, 65: 611-621.

16. Custers E.J., Regehr, G., Norman, G.R. (1996). Mental Representations of Medical Diagnostic Knowledge: A Review. Academic Medicine; 71: Suppl: S55-S61.

17. Nendaz, M.R., Bordage, G. (2002). Promoting diagnostic problem representation. Medical Education, 36: 760-766.

18. Chang, R.W., Bordage, G., Cornell, K.J., (1998). The importance of early problem representation during case presentations. Academic Medicine, 73: Suppl: S109-S111.

19. Norman, G.R., Brooks, L.R. (1997). The nonanalytical basis of clinical reasoning. Adv Health Sci Educ Theory Pract; 2(2): 173-84. 